\title{
Conformational and Compositional Tuning of Phenanthrocarbazole- Based Dopant-Free Hole-Transport Polymers Boosting the Performance of Perovskite Solar Cells
}

\author{
Zhaoyang Yao, ${ }^{\ddagger}$ Fuguo Zhang, ${ }^{\ddagger}$ Yaxiao Guo, ${ }^{\ddagger}$ Heng Wu, Lanlan He, Zhou Liu, Bin Cai, Yu Guo, \\ Calvin J. Brett, Yuanyuan Li, Chinmaya Venugopal Srambickal, Xichuan Yang, Gang Chen, \\ Jerker Widengren, Dianyi Liu, James M. Gardner, Lars Kloo, and Licheng Sun*
}

Cite This: J. Am. Chem. Soc. 2020, 142, 17681-17692

Read Online

ABSTRACT: Conjugated polymers are regarded as promising candidates for dopantfree hole-transport materials (HTMs) in efficient and stable perovskite solar cells (PSCs). Thus far, the vast majority of polymeric HTMs feature structurally complicated benzo[1,2- $\left.b: 4,5-b^{\prime}\right]$ dithiophene (BDT) analogs and electron-withdrawing heterocycles, forming a strong donor-acceptor $(\mathrm{D}-\mathrm{A})$ structure. Herein, a new class of phenanthrocarbazole (PC)-based polymeric HTMs (PC1, PC2, and PC3) has been synthesized by inserting a PC unit into a polymeric thiophene or selenophene chain with the aim of enhancing the $\pi-\pi$ stacking of adjacent polymer chains and also to efficiently interact with the perovskite surface through the broad and planar conjugated backbone of the PC. Suitable energy levels, excellent thermostability, and humidity resistivity together with remarkable photoelectric properties are obtained via meticulously tuning the conformation and elemental

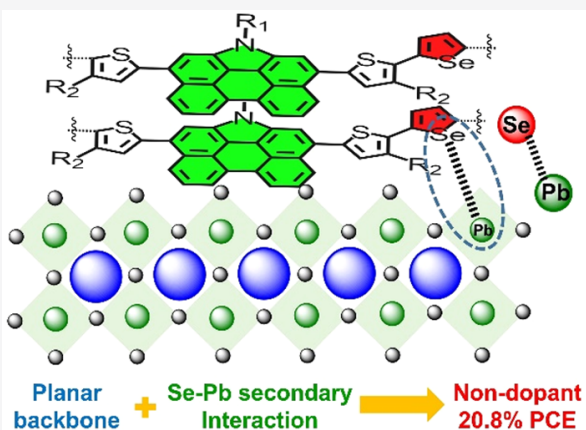
composition of the polymers. As a result, PSCs containing PC3 as dopant-free HTM show a stabilized power conversion efficiency (PCE) of $20.8 \%$ and significantly enhanced longevity, rendering one of the best types of PSCs based on dopant-free HTMs. Subsequent experimental and theoretical studies reveal that the planar conformation of the polymers contributes to an ordered and face-on stacking of the polymer chains. Furthermore, introduction of the "Lewis soft" selenium atom can passivate surface trap sites of perovskite films by $\mathrm{Pb}-\mathrm{Se}$ interaction and facilitate the interfacial charge separation significantly. This work reveals the guiding principles for rational design of dopant-free polymeric HTMs and also inspires rational exploration of small molecular HTMs.

\section{INTRODUCTION}

Perovskite solar cells (PSCs) have gone through an unprecedented increase in power conversion efficiency (PCE) from a mere $3.8 \%$ to $25.2 \%$ in the past decade due to the excellent light-harvesting capacity and unrivalled chargecarrier diffusion length of organic-inorganic hybrid lead halide perovskites. $^{1-9}$ In spite of the rapidly surging PCE, PSCs have also caused extensive scientific studies because of their exceptional photoelectric character, facile processability, and promising commercial potential. ${ }^{10-12}$ Recently, perovskite/Si tandem devices surpassed crystalline Si photovoltaics, displaying an exciting PCE of $29.1 \%$, greatly boosting PSCs toward the ultimate aim of economic feasibility. ${ }^{9}$ However, there are still several hurdles on the way to commercial application, for example, the inherent thermal and humidity instability due to the ionic nature of the perovskite crystals and the interstitial degradation caused by adverse interfacial reactions. ${ }^{13-15}$ Therefore, massive research has focused on prolonging the lifespan of PSCs. ${ }^{16-22}$ In particular, attenuation of PCE induced by dopants in charge-transporting layers has received special concern. ${ }^{8}$ In a typical n-i-p device architecture, hole- transport materials (HTMs) play an imperative role in efficient hole extraction from the perovskite layer and transport to the positive electrode, preventing the moisture-sensitive perovskite material from directly touching the water molecules in ambient atmosphere. Therefore, rational selection of HTMs is very crucial for boosting the PCE and device longevity of PSCs. Thus far, a large number of organic small molecules, conjugated polymers, as well as inorganic materials have been explored as HTMs for efficient PSCs. ${ }^{23-31}$ Among them, $2,2^{\prime}, 7,7^{\prime}$-tetrakis $(N, N$-di- $p$-methoxyphenylamine)-9,9' -spirobifuorene (spiro-OMeTAD) $)^{32,33}$ usually serves as the state-ofthe-art HTM for the benchmarking of PSCs containing new materials. ${ }^{7,34}$ Nevertheless, despite the unique success of spiro$\mathrm{OMeTAD}$, the material still suffers from unaffordable cost and

Received: August 3, 2020

Published: September 14, 2020 
tedious synthesis, probably disqualifying it from large-scale fabrication of PSCs. Even more fatal is that the steric hindrance of the spiro center gives rise to a large resistivity and low charge mobility. Therefore, introduction of proper dopants becomes necessary to increase the charge transport capacity of spiro-OMeTAD. ${ }^{35-38}$ However, the basic problem is that the widely utilized dopants, such as lithium bis(trifluoromethanesulfonyl)imide (LiTFSI), tert-butylpyridine (TBP), and cobalt metal complexes, all are hygroscopic or volatile. ${ }^{39,40}$ Moreover, migration and relocation of the noncovalently bonded dopants usually cause morphology deterioration of the HTM layers. ${ }^{41-43}$ All of the adverse aspects mentioned above will accelerate device deterioration significantly under a practical application environment, making the doping strategy inappropriate for production and industrialization. Therefore, a large amount of research has been devoted to exploring next-generation HTMs with the aim of improving the stability and economic feasibility of PSCs avoiding the need for dopants. ${ }^{44-46}$ Thus far, very few of the top class PSCs containing dopant-free HTMs have been reported.

Conjugated polymers, characteristic of excellent and tunable physicochemical properties, have been widely applied in organic optoelectronic devices. Compared to some other HTMs used in PSCs, such as small molecules or inorganic materials, polymeric HTMs possess several advantages involving excellent carrier mobility, thermal and optical stability, solution processability, film-forming ability, and potential industrial production through roll-to-roll printing technologies. ${ }^{47-49}$ Thus far, several dopant-free polymeric HTMs have been applied in PSCs and contributed to relatively high PCEs and significantly enhanced device stability. ${ }^{50-57}$ However, nearly all known materials feature a strong donoracceptor $(\mathrm{D}-\mathrm{A})$ structure inherited from the polymeric donors in bulk heterojunction organic solar cells (OSCs). Polymeric donors in OSCs should extract and conduct holes efficiently and at the same time act as powerful light-harvesting materials to absorb sufficient photons from sunlight. ${ }^{58,59}$ However, HTMs in PSCs are preferably colorless and photochemically inert because the perovskite films themselves provide saturated absorption of visible light under irradiance of simulated AM $1.5 \mathrm{G}$ sunlight $\left(100 \mathrm{~mW} \mathrm{~cm} \mathrm{~cm}^{-2}\right)$. Thus, the strong electron acceptors, which are structurally complicated and cost driving, are unnecessary in HTMs for PSCs. Bearing this in mind, we simply polymerized the widely used benzo[1,2-b:4,5- $\left.b^{\prime}\right]$ dithiophene (BDT) unit with thiophene and synthesized the low-cost P3 (Figure S1) as a dopant-free HTM in a recent study from our lab. ${ }^{60}$ P3-based PSCs provide an exceptional stabilized PCE of $20.3 \%$, which is comparable to control devices based on doped spiro-OMeTAD. This work demonstrated that polymers without strong $\mathrm{D}-\mathrm{A}$ structures could also work efficiently as HTMs in PSCs. However, the BDT analogs, which involve multiple reaction steps and dangerous reagents $(n-\mathrm{BuLi}, \mathrm{LDA}$, etc.) during the tedious synthesis, are still indispensable for the highly efficient HTMs in PSCs or even donor polymers in OSCs.

Perylene and its derivatives are excellent candidates for functional materials in many fields. ${ }^{61,62}$ In this work, a series of non-BDT polymers, PC1, PC2, and PC3, was designed and synthesized through incorporating the large heteroatomcontaining $\mathrm{PAH}$ unit phenanthrocarbazole (PC) into polymeric thiophene or selenophene chains. The obtained polymers feature suitable energy levels, excellent thermo- stability and humidity resistivity, exceptional film-forming ability, and remarkable photoelectric properties as dopantfree HTMs in PSCs. Consequently, PSCs containing PC3 as the dopant-free HTMs showed a stabilized PCE of $20.8 \%$ and significantly enhanced stability. Experimental and theoretical studies reveal that the planar conformation of the polymers will facilitate the more ordered and face-on stacking of the polymer strands. Furthermore, introduction of the "Lewis soft" selenium element passivates the surface trap sites on the surface of perovskite films by a $\mathrm{Pb}-\mathrm{Se}$ bond. The guiding principles for rational design of dopant-free polymeric HTMs are illustrated in this work.

\section{RESULTS AND DISCUSSION}

Figure 1 displays the molecular structures and design strategy of the three polymers PC1, PC2, and PC3. We first inserted

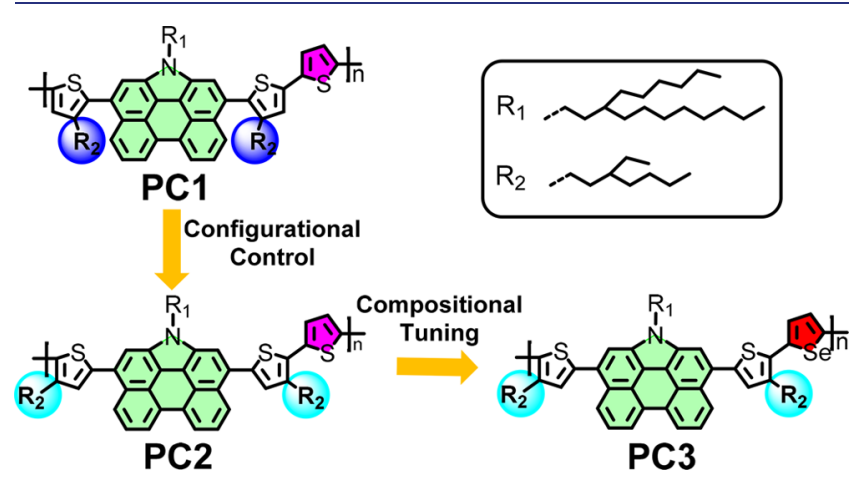

Figure 1. Molecular structures and design strategy of the three polymers PC1, PC2, and PC3.

PC into the polymeric thiophene chain with the aim of enhancing the $\pi-\pi$ stacking of the polymer chains and also to improve interaction with the perovskite surface through the broad and planar conjugated backbone of the PC unit. On the premise of strong $\pi-\pi$ stacking, several bulky and hydrophobic aliphatic chains were grafted on the rigid backbone to improve the solubility and humidity resistivity of the polymers as HTMs. From PC1 to PC2, we used configurational control by changing the position of the side chains on the thiophene units. In this way, PC2 mitigates the significant steric hindrance caused by the $\mathrm{R}_{2}$ groups and the bulky PC unit, maintaining a more planar configuration. The relatively planar geometry will facilitate the $\pi-\pi$ stacking between adjacent polymer chains and most likely increase the hole mobility in the resulting HTM films. Further compositional tuning was made in PC3 by introducing selenium, which may further improve the hole mobility through its larger polarizability. At the same time, selenium may passivate the sites of insufficiently coordinated lead ions.

Scheme 1 illustrates the synthetic routes to the three new polymers. The detailed synthetic procedures and characterization data of the new compounds are given in the Supporting Information. For the PC1 polymer's synthesis, the reported 3,10-dibromo-1-(2-hexyldecyl)-1H-phenanthro[1,10,9,8cdefg]carbazole $(\mathbf{1})^{63}$ was treated with $n$-butyllithium in an inert atmosphere to get a rapid halogen-lithium exchange and then transformed into its borate $\mathbf{2}$ by adding 2-isopropoxy4,4,5,5-tetramethyl-1,3,2-dioxaborolane. The obtained 2 was cross-coupled with 2-bromo-3-(2-ethylhexyl)thiophene by a Suzuki reaction to generate the intermediate 3 , which was 
Scheme 1. Synthetic Routes to PC-Based Polymers ${ }^{a}$

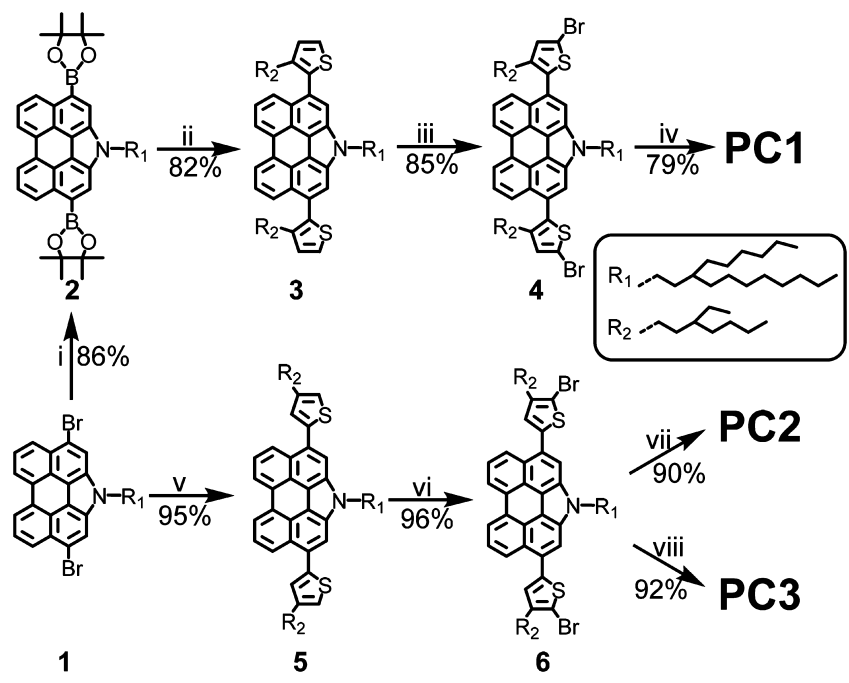

${ }^{a}$ Reagents and conditions: (i) $n$-BuLi, THF, $-78{ }^{\circ} \mathrm{C}, 1 \mathrm{~h}$; then 2 isopropoxy-4,4,5,5-tetramethyl-1,3,2-dioxaborolane, $-78{ }^{\circ} \mathrm{C}$ to $\mathrm{RT}, 1$ h; (ii) 2-bromo-3-(2-ethylhexyl)thiophene, $\mathrm{Pd}\left(\mathrm{PPh}_{3}\right)_{4}, \mathrm{~K}_{2} \mathrm{CO}_{3}$, toluene $/ \mathrm{H}_{2} \mathrm{O} /$ ethanol $(1 / 1 / 0.2, \mathrm{v} / \mathrm{v} / \mathrm{v})$, reflux, overnight; (iii) NBS, THF, $0{ }^{\circ} \mathrm{C}$ to $\mathrm{RT}, 2 \mathrm{~h}$; (iv) 2,5-bis(trimethylstannyl)thiophene, $\mathrm{Pd}_{2}(\mathrm{dba})_{3}, \mathrm{P}(o \text {-tol })_{3}$, chlorobenzene, reflux, $\left.72 \mathrm{~h} ; \mathrm{v}\right)$ (4-(2ethylhexyl)thiophen-2-yl)trimethylstannane, $\mathrm{Pd}_{2}(\mathrm{dba})_{3}, \mathrm{P}(o \text {-tol })_{3}$, toluene, reflux, $6 \mathrm{~h}$; (vi) NBS, THF, $0{ }^{\circ} \mathrm{C}$ to RT, $2 \mathrm{~h}$; (vii) 2,5bis(trimethylstannyl)thiophene, $\mathrm{Pd}_{2}(\mathrm{dba})_{3}, \mathrm{P}(o \text {-tol })_{3}$, chlorobenzene, reflux, $72 \mathrm{~h}$; (viii) 2,5-bis(trimethylstannyl)selenophene, $\mathrm{Pd}_{2}(\mathrm{dba})_{3}$, $\mathrm{P}(\mathrm{o} \text {-tol })_{3}$, chlorobenzene, reflux, $72 \mathrm{~h}$.

further brominated using the mild reagent NBS to give the monomer 4 in good yield. Finally, polymerization of compound 4 was achieved with 2,5-bis(trimethylstannyl)- thiophene through a Stille coupling reaction, affording the PC1 polymer. The syntheses of PC2 and PC3 started with the cross-coupling reaction between compound 1 and (4-(2ethylhexyl)thiophen-2-yl)trimethylstannane to afford $\mathbf{5}$ in an excellent yield. Thereafter, compound $\mathbf{5}$ was brominated in the presence of NBS to quantitatively yield the monomer 6 , which was further polymerized with 2,5-bis(trimethylstannyl)thiophene and 2,5-bis(trimethylstannyl)selenophene to produce PC2 and PC3 in an excellent yield, respectively.

The molecular weights of the three polymers were estimated by gel permeation chromatography (GPC). The average molecular weights $\left(M_{\mathrm{n}}\right)$ were calculated as about 30,39 , and $40 \mathrm{kDa}$ with polydispersity indexes (PDI) of 1.12, 1.20, and 1.19 for PC1, PC2, and PC3, respectively. It is well known that diverse molecular weights may give rise to differences in light absorption, hole mobility, thermal stability, and photovoltaic properties of the polymers. In order to precisely reveal the molecular structure-dependent physicochemical properties, we first investigated the repeat units of the polymers showing welldefined structures. This allows a more rational comparison between the structures and the properties. As shown in Figure 2a, the polymer segments PC1-M, PC2-M, and PC3-M were constructed by shortening the aliphatic chains of the polymers. The synthetic routes to PC1-M, PC2-M, and PC3-M and the detailed procedures are presented in Scheme S2 in the Supporting Information. Density functional theory (DFT) calculations at the level of B3LYP/6-31G(d,p) generated optimized geometries and energy levels of the frontier molecular orbitals for these three polymer fragments. A large dihedral angle of $64^{\circ}$ between the thiophene and the PC unit can be observed in PC1-M (Figure 2b), which should be attributed to the large steric hindrance caused by the substituent groups on the thiophene unit and the bulky
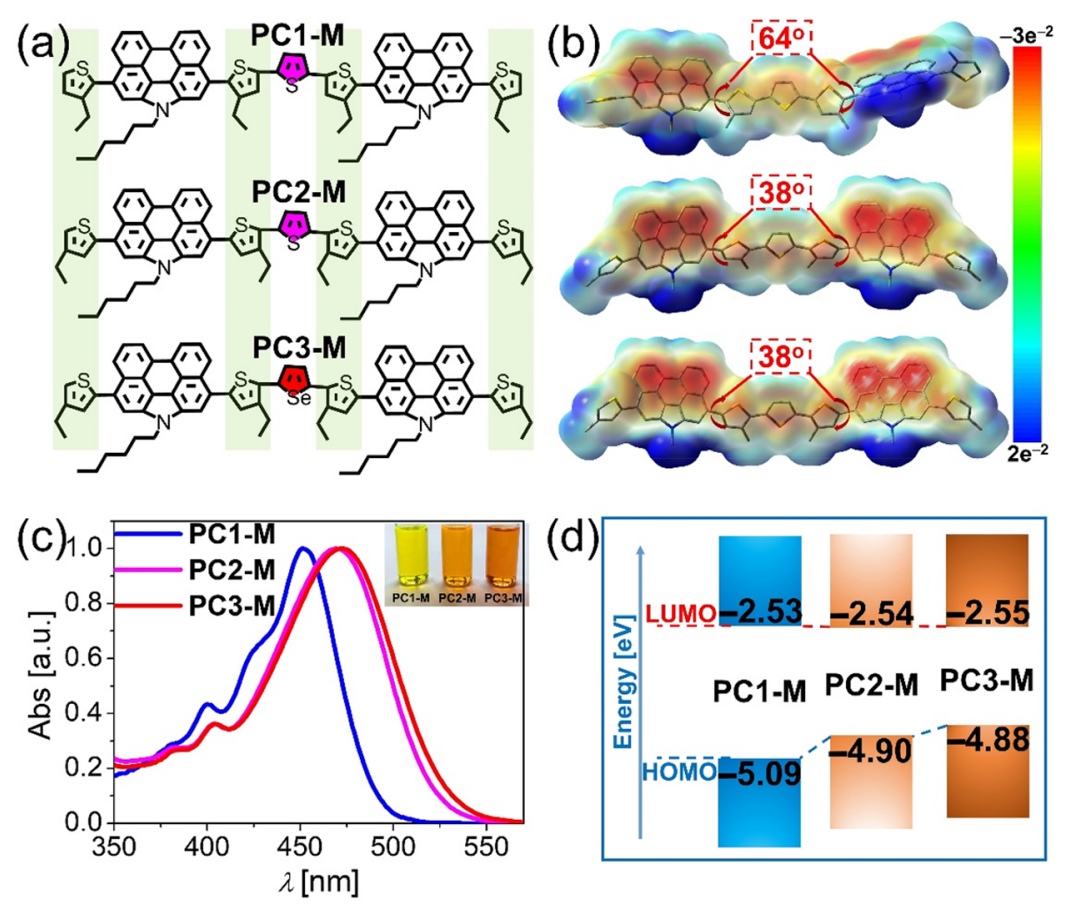

Figure 2. (a) Molecular structures of fractions (PC1-M, PC2-M, and PC3-M) of the PC-based polymers. (b) Optimized geometries and electrostatic surface potential maps (ESPs) obtained by density functional theory (DFT) calculations. Large aliphatic groups were substituted with methyl groups to accelerate the computations. Dihedral angles between the PC units and neighboring thiophenes are highlighted. (c) normalized electronic absorption spectra in $20 \mu \mathrm{M}$ THF solution. (d) Energy levels derived from solution cyclic voltammetry and optical band gaps. 

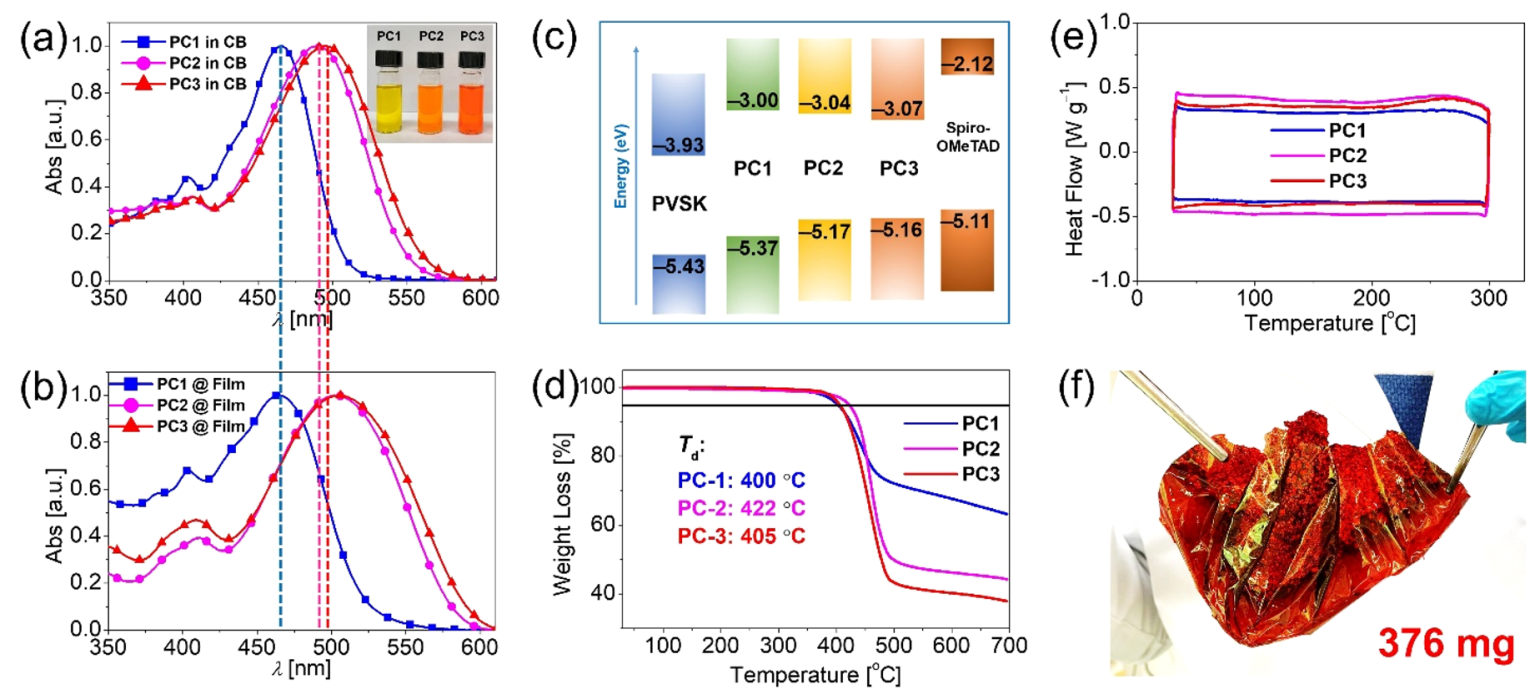

Figure 3. (a) Normalized electronic absorption spectra of the polymers PC1, PC2, and PC3 in chlorobenzene (CB) solutions. (b) Normalized electronic absorption spectra of polymers in the solid films. (c) Energy levels of polymer films derived from CV and optical band gaps. (d) TGA traces of the polymers. (e) DSC traces of the polymers. (f) Obtained film of PC3 polymer.

backbone of PC. ${ }^{64}$ Conformational effects by changing the grafting site of the alkyl chain to the other side of the thiophene unit mitigates the steric hindrance, which contributes to a more planar backbone of PC2-M with respect to PC1-M. As might be expected, compositional tuning by introducing selenium into PC3-M in place of sulfur does not influence the molecular geometry significantly. The electrostatic surface potential maps (ESPs) of PC1-M, PC2-M, and PC3-M demonstrate the electron-rich property of the PC units. Consequently, the results from the theoretical calculations show relatively high energy levels of the highest occupied molecular orbitals (HOMOs) of $-4.87 \mathrm{eV}$ for PC1M, $-4.73 \mathrm{eV}$ for PC2-M, and $-4.70 \mathrm{eV}$ for PC3-M, upshifting with the structural modifications (Figure S2). However, the lowest unoccupied molecular orbital (LUMO) energy levels are located at $-2.01 \mathrm{eV}$ for PC1-M, $-2.07 \mathrm{eV}$ for PC2-M, and $-2.10 \mathrm{eV}$ for PC3-M, giving rise to a stepwise smaller energy gap of $2.86 \mathrm{eV}$ for PC1-M, $2.66 \mathrm{eV}$ for PC2-M, and $2.60 \mathrm{eV}$ for PC3-M. It is thus plausible to observe the red-shifted maximum absorption wavelength $\left(\lambda_{\mathrm{MAX}}^{\mathrm{Sol}}\right)$ from PC1-M to PC3-M in accordance with the electronic absorption spectra of the three molecules simulated by time-dependent DFT calculations (Figure S3). The S0 $\rightarrow$ S1 vertical electronic transitions to the LUMO for three molecules occur predominantly from the HOMO, demonstrating a clear intramolecular charge-transfer character. The normalized electronic absorption spectra in THF solvent are shown in Figure 2c. $\lambda_{\mathrm{MAX}}^{\mathrm{Sol}}$ for PC2-M and PC3-M are 469 and $473 \mathrm{~nm}$, respectively, about $20 \mathrm{~nm}$ red shifted with respect to $451 \mathrm{~nm}$ for PC1-M. A similar trend can be also observed from the absorption spectra in the state of thin films of the molecules (Figure S4). The red-shifted absorption should be mainly attributed to the up-shifted HOMO energy levels of the constituent molecules. Cyclic voltammetry (CV) was employed to estimate the experimental energy levels of the molecules (Figure S5). The HOMO energy levels were estimated by $E=-4.8-e E_{\text {onset }}$ where $E_{\text {onset }}$ is the onset redox potential of a ground-state molecule in THF, being $-5.09 \mathrm{eV}$ for PC1-M, $-4.90 \mathrm{eV}$ for PC2-M, and $-4.88 \mathrm{eV}$ for PC3-M (Figure 2d). As listed in Table S1, the relative alignment of the energy levels as well as the red-shift tendency of $\lambda_{\text {MAX }}^{\text {Sol }}$ are in accordance with the results from the theoretical calculations.

From molecular fragments to the polymers, we observed that all three polymers show very good solubility in several commonly used solvents, such as chloroform, toluene, and chlorobenzene, facilitating their later solution-based process for PSCs fabrication. Figure $3 a$ and $3 b$ shows the electronic absorption spectra of the three polymers dissolved in chlorobenzene and as solid thin films, respectively. In accordance with the absorption features noted for the PC-M fragment molecules, the spectra of PC2 and PC3 are bathochromically shifted by nearly $30 \mathrm{~nm}$ as compared to that of PC1, which can be attributed to their more planar structures. It is also notable that no obvious difference is observed regarding the maximum absorption wavelength of PC1 from solution to a solid thin film; however, an approximate $10 \mathrm{~nm}$ red shift can be observed for PC2 and PC3. This might imply that J-type aggregates, which can push the absorption toward low energies, may be formed by PC2 and PC3 because of their relatively planar backbones, suggesting a difference in stacking with respect to PC1. ${ }^{65}$ Comparing with PC2, the absorption of PC3 demonstrated a slight red shift of 3 and $6 \mathrm{~nm}$ in solutions and as solid films, respectively, which is expected to arise from the more electronrich property of the Se atom than the $S$ atom. The optical band gaps of the three polymers can be estimated as $2.37,2.13$, and $2.09 \mathrm{eV}$, respectively. Figure $3 \mathrm{c}$ shows the relative alignment of the energy levels of the frontier molecular orbitals as derived from the CVs of the solid thin films (Figure S6) and optical band gaps. The HOMO energy levels are determined to be $-5.37 \mathrm{eV}$ for PC1, $-5.17 \mathrm{eV}$ for PC2, and $-5.16 \mathrm{eV}$ for PC3, and the LUMO energy levels are estimated to be $-3.00 \mathrm{eV}$ for PC1, $-3.04 \mathrm{eV}$ for PC2, and $-3.07 \mathrm{eV}$ for PC3. All three polymers exhibit HOMOs and LUMOs located in a suitable energy range when comparing with the energy levels of the perovskite material used. This enables the polymers to efficiently extract holes from the perovskite film and simultaneously block the reverse electron flow from the perovskite to the metal back contact. Furthermore, we 
performed thermal gravimetric analysis (TGA) and differential scanning calorimetry (DSC) to monitor the phase stability of the polymers under thermal stress. As revealed in Figure $3 \mathrm{~d}$ and $3 \mathrm{e}$, the decomposition temperatures, defined as having lost $5 \%$ weight, are 400,422 , and $405{ }^{\circ} \mathrm{C}$ for PC1, PC2, and PC3, respectively. No obvious peaks can be noticed in the DSC curves at lower temperatures, suggesting that the polymers are thermally stable without detectable phase transitions up to 300 ${ }^{\circ} \mathrm{C}$. All of the polymers thus display low crystallinity and high thermal stability in the film state, which is a prerequisite for durable device operation. The PC3 polymer forms an exceptionally compact film, which can be attributed to the strong $\pi-\pi$ stacking of the broad and planar conjugated backbone of PC (Figure 3f). The excellent film-forming ability of the polymers can facilitate effective coverage of the perovskite surface even at a low amount of material. The detailed parameters of the optical, thermal, and electrochemical properties are listed in Table S2.

An adequate charge-transporting ability is vital for the polymeric materials to become useful as HTMs in PSCs, and therefore, hole-only devices with the structure of ITO/ poly(3,4-ethylenedioxythiophene)-poly(styrenesulfonate) (PEDOT:PSS)/HTM/Au were constructed to measure the hole mobility of the HTMs by utilizing the space charge-limited current (SCLC) method. As displayed in Figure 4a, the estimated hole mobilities $\left(\mu_{\mathrm{h}}\right)$ of PC2 and PC3 are $7.3 \times 10^{-4}$ and $8.1 \times 10^{-4} \mathrm{~cm}^{2} \mathrm{~V}^{-1} \mathrm{~s}^{-1}$, respectively, demonstrating over 1 order of magnitude higher mobility than that of PC1, being 4.2 $\times 10^{-5} \mathrm{~cm}^{2} \mathrm{~V}^{-1} \mathrm{~s}^{-1}$. The slightly higher $\mu_{\mathrm{h}}$ of PC3 with respect to PC2 can be probably rationalized as an effect of the incorporated selenium atom in the structures given the only structural change is the replacement of sulfur with selenium. However, the dramatic differences of $\mu_{\mathrm{h}}$ between the two "isomers" PC1 and PC2 are expected to be correlated to the significantly different geometries of the respective polymeric backbones (twisted for PC1 and planar for PC2).

In order to gain insight into the underlying correlation between $\mu_{\mathrm{h}}$ and the molecular packing, grazing-incidence wideangle X-ray scattering (GIWAXS) experiments were carried out (Figure S7) ${ }^{66,67}$ As observed from Figure 4b and 4c, PC2 and PC3 mainly feature a typical face-on orientation as discerned from the apparent peaks in the in-plane direction $\left(q_{\mathrm{r}}\right.$ $\left.\approx 0.29 \AA^{-1}\right)$ and the out-of-plane direction $\left(q_{z} \approx 1.5 \AA^{-1}\right)$. In contrast, the PC1 polymer displays a much weaker peak at $q_{z} \approx$ $1.4 \AA^{-1}$ along the out-of-plane direction and an unambiguous lattice plane (100) in the in-plane direction, suggesting a more edge-on orientation of molecular stacking. The edge-on orientation is expected to impede efficient charge transport in the vertical diode architecture of solar cells. ${ }^{68}$ These results are in accordance with the lower hole mobility observed for PC1 film as compared to that of PC2 and PC3. Furthermore, PC2 and PC3 display a shorter $\pi-\pi$ stacking distance of $\sim 4.1$ $\AA$ with respect to that of $4.5 \AA$ for PC1, further confirming that the planar polymer backbone favors a more compact packing of the polymer chains through secondary interactions. The closer stacking may enhance an effective overlap of orbitals between the neighboring polymer strands, facilitating charge mobility. ${ }^{69}$ Meanwhile, the optimized stacking models by piling up three layers of polymers were obtained by the DFT calculations (Figure 4d). As might be expected, PC2 and PC3 exhibit a more ordered molecular packing pattern than PC1 due to their more planar conformations. This is in good agreement with the results from the experiments. To
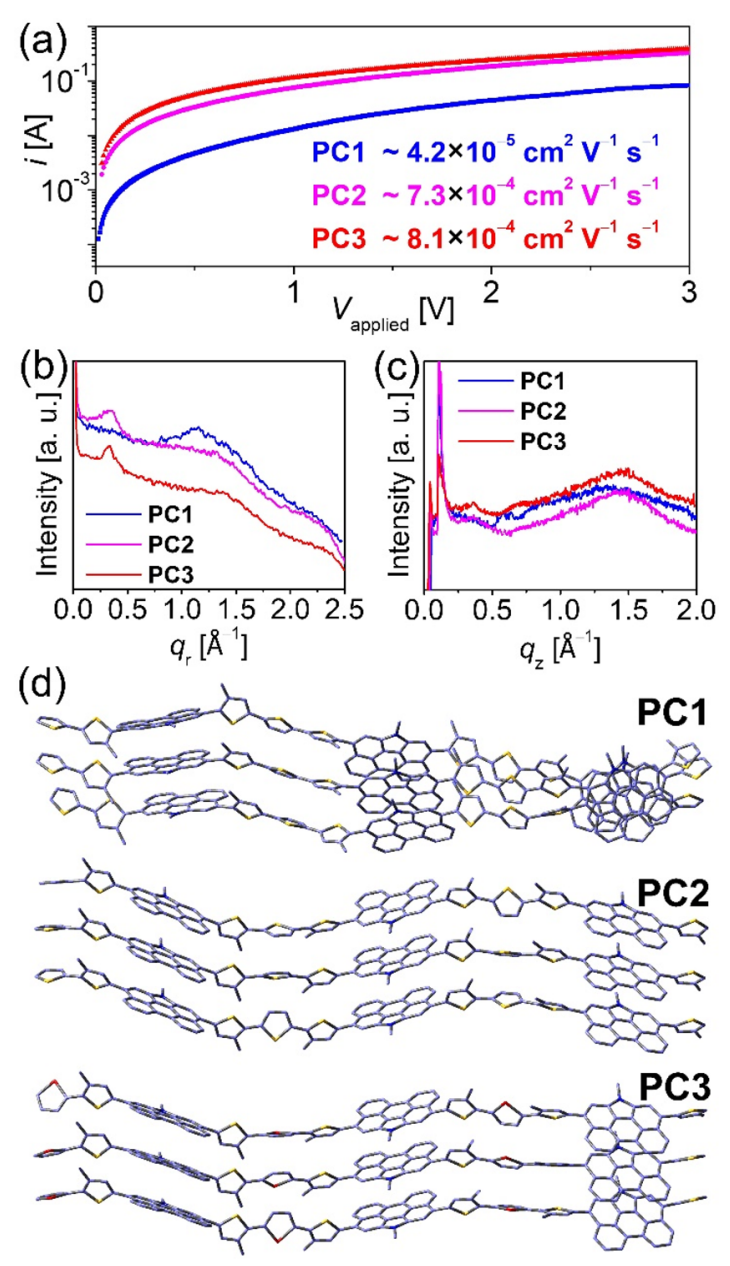

Figure 4. (a) Hole mobilities of the three polymers derived from SCLC measurements. (b) In-plane and (c) out-of-plane line cuts of the 2D GIWAXS traces for the three polymers on silicon substrates. (d) Optimized stacking models obtained by DFT calculations.

summarize, all of the above experimental and theoretical modeling results are in good agreement with the lower vertical $\mu_{\mathrm{h}}$ of PC1 as compared to PC2 and PC3. Moreover, molecular stacking effects on the absorption differences of the three polymers going from solutions to thin solid films emerge as a logical consequence. The face-on orientation as well as the sequentially compact polymer stacking in PC2 and PC3 are expected to result in better performance of the resulting PSC devices as compared to those based on PC1. In order to investigate the film morphology of the polymers when coated on top of the perovskite films, field emission scanning electron microscopy (FE-SEM) and atomic force microscopy (AFM) were performed. Figure S8 displays the SEM images of the noncovered perovskite surface and with the three polymers capping perovskite films. The excellent film-processing ability attributed to the planar backbones in PC2 and PC3 results in uniform films that clad the surface of the perovskite layers perfectly. AFM images were further analyzed to extract morphology information on the perovskite films covered by the different polymers on a nanoscale level. As displayed in Figure S9, the root-mean-square (RMS) roughness amounts of PC1, PC2, and PC3 were estimated to be 13, 12, and $11 \mathrm{~nm}$, respectively, significantly smaller than that of neat perovskite film (20 nm; the estimated statistical error of the roughness is less than $0.5 \mathrm{~nm}$ ). The complete and uniform coverage by the 

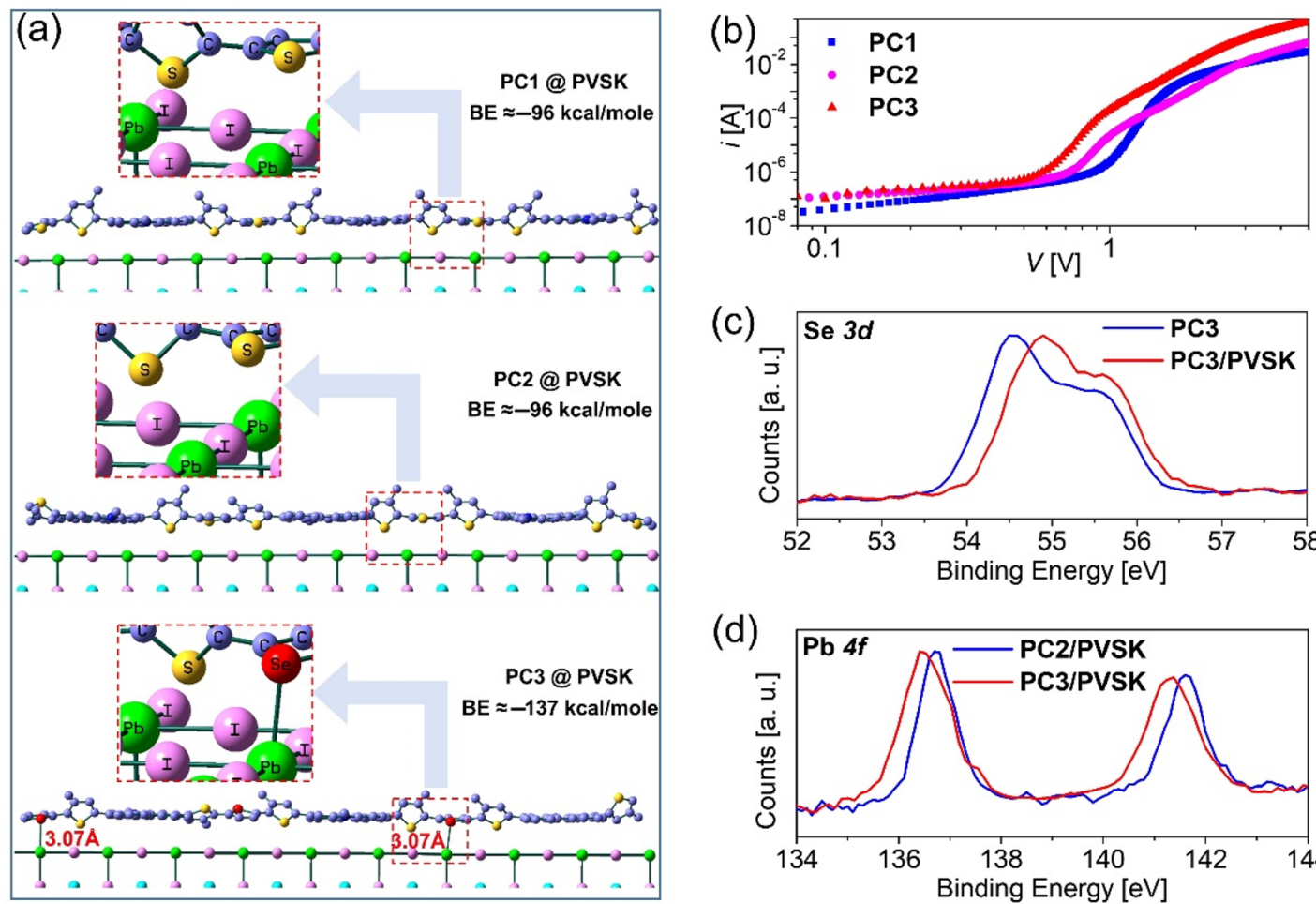

(c)

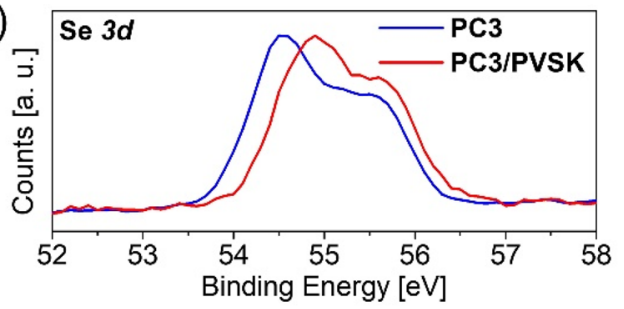

(d)

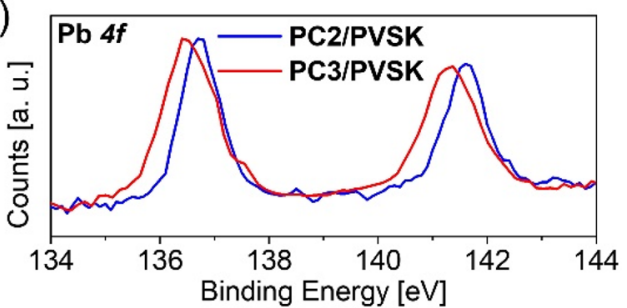

Figure 5. (a) Theoretically modeled stacking patterns of the PC polymers on top of a perovskite (PVSK) surface. Binding energies (BE) between the polymers and the perovskite surface are given. (b) Trap state densities of the PC polymers coating perovskite films. (c) XPS signals of Se 3d from a pristine PC3 film and from a PC3-coated perovskite film. (d) XPS signals of Pb 4f from PC2-and PC3-coated perovskite films.

polymers prevents the perovskite layer from direct contact with the counter electrode materials and may thus significantly reduce undesired shunt losses in the resulting PSCs.

Considerations concerning the perovskite surface passivation by electron-rich heteroatoms were behind our rational inclusion of abundant nitrogen, sulfur, and selenium elements into the new PC-based polymeric HTMs. Thus far, the widely utilized passivators have been confined to the oxygen-, nitrogen-, and sulfur-containing compounds. ${ }^{7,70}$ However, selenium-involved passivators are rarely reported in the literature. The empirical covalent radius and calculated atomic radius of selenium are 115 and $103 \mathrm{pm}$, respectively, clearly larger than those of 100 and 88 pm for sulfur. ${ }^{71,72}$ Therefore, the larger atomic radius and "Lewis softer" selenium atom represents a characteristic example of a highly electron-rich, easy polarizable, and relatively large element. Therefore, selenium has properties that may offer efficient surface passivation to stabilize coordinatively unsaturated lead ion at the perovskite-HTM interface. In order to investigate the secondary interaction between polymeric HTMs and the perovskite films, DFT calculations were conducted by positioning three repeat segments of polymeric HTMs on the top of a perovskite crystal model with subsequent constraint optimization. As demonstrated in Figure 5a and Figure S10, all three polymeric HTMs interact tightly with the perovskite surface through the rigid and bulk conjugated plane of PC. This gives support to the strategy of our design of introducing large $\mathrm{PAH}$ units to enhance the interfacial packing between the perovskite surface and the HTMs. It is worth noting that the selenium atoms in PC3 are prone to approach lead sites at the perovskite surface, forming weak but specific interactions characterized by a bond length of $3.07 \AA$, resulting in a binding energy of $-137 \mathrm{kcal} / \mathrm{mol}$ between the PC3 segment and perovskite layer, which is much larger than that of $-96 \mathrm{kcal} / \mathrm{mol}$ obtained for the PC1 and PC2 systems. The tighter packing will lead to a larger orbital overlap at the perovskite/HTM interface and consequently facilitate hole extraction. The trap state sites on the surface of the perovskite film can be partially passivated by Lewis bases, such as the electron-rich atoms being part of the HTMs as discussed above, indicating the extent of passivation by the HTMs. Thereby, we made specific devices with an architecture of ITO/PEDOT:PSS $/ \mathrm{MAPbI}_{3} / \mathrm{HTM} / \mathrm{Au}$ to estimate the trapstate densities directly and further evaluate the passivation effects of the three HTMs (Figure 5b). The trap-filling-limited voltages $\left(V_{\mathrm{TFL}}\right)$ of the three dopant-free PC1-, PC2-, and PC3coated perovskite films were obtained as $0.91,0.72$, and $0.54 \mathrm{~V}$, respectively. The corresponding trap-state densities were determined to $1.55 \times 10^{16}, 1.21 \times 10^{16}$, and $9.2 \times 10^{15}$ $\mathrm{cm}^{-3}$, respectively. The significantly lower trap-state density of the perovskite surfaces capped by PC3 should be attributed to the higher passivation effect of selenium. This is in good agreement with the results from our DFT modeling. The lower density of trap states for PC3-coated perovskite films will be expected to generate fewer recombination centers and thus afford a better photovoltaic performance. X-ray photoelectron spectroscopy (XPS) was carried out to monitor the energy change of characteristic peaks caused by selenium passivation (Figure $5 \mathrm{c}$ and $5 \mathrm{~d}$ ). The Se $3 \mathrm{~d}$ peak of PC3-coated perovskite films is located at $54.9 \mathrm{eV}$, shifting to a higher binding energy with respect to the $54.6 \mathrm{eV}$ for a pristine PC3 film. This indicates a direct interaction between selenium in PC3 and the perovskite surface. The symmetric peaks that appear at 136.7 and $141.6 \mathrm{eV}$ of PC2-capped perovskite films should be ascribed to the $\mathrm{Pb} 4 \mathrm{f}$ core level. The spin-orbit split of $4.8 \mathrm{eV}$ between the $4 f_{7 / 2}$ and the $4 f_{5 / 2}$ peaks is in good accordance 
with reported values. ${ }^{73}$ However, for the PC3-capped perovskite films, these peaks appear at 136.4 and $141.3 \mathrm{eV}$, showing a $\sim 0.3 \mathrm{eV}$ shift toward the low-binding energy direction. These shifts should be attributed to the $\mathrm{Pb}-\mathrm{Se}$ interaction mediated by the PC3 polymer and perovskite surface.

Steady-state PL spectra (Figure 6a) and time-resolved PL decay (TRPL) traces (Figure $6 \mathrm{~b}$ ) were recorded to elucidate
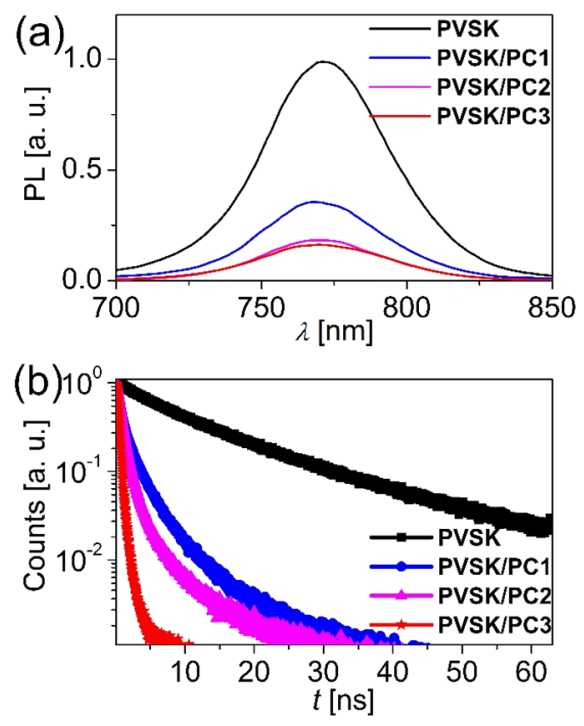

Figure 6. (a) Steady-state photoluminescence (PL) spectra and (b) time-resolved PL decay traces of the perovskite films (PVSK) and HTM-capped perovskite films.

the interfacial charge transfer between the three polymers and the perovskite films. Capping with diverse HTMs will evoke PL quenching of the perovskite films, indicating hole extraction at the energy-offset HTM/perovskite interface. With respect to PC1-capped perovskite film, PC2- and PC3-capped ones display a clearly more efficient depression of the perovskite PL intensity. This may be partly caused by the larger hole mobility and upshifted HOMO energy levels of these polymers which will provide a larger driving force for hole collection. The TRPL decay traces can be modeled by a biexponential equation, and the obtained parameters are given in Table S3. The results are in good agreement with the steady-state PL spectra, where a dramatically reduced PL lifetime can be observed when the pure perovskite film is capped by the polymers. Among the polymers, the PC3 system demonstrates a reduced PL lifetime with respect to the other two polymers, suggesting a more efficient hole extraction from the perovskite film to the HTM layer.

In order to explore application of the polymers as dopantfree HTMs, PSCs with the regular architecture of glass/FTO (flourine-doped tin oxide)/mesoporous $\mathrm{SnO}_{2} /$ perovskite/ HTM/Au were assembled (Figure $7 \mathrm{a}$ ). ${ }^{74}$ All of the polymers were applied as HTMs without any dopants in this work. The detailed fabrication of PSCs is described in the Supporting Information. We first investigated the device performance related to the HTM film thickness by adjusting the concentrations of the HTM precursors from 3 to $10 \mathrm{mg} / \mathrm{mL}$ in chlorobenzene, and the obtained photovoltaic parameters are displayed in the Supporting Information (Figure S11 and Table S4). All three polymers showed the best device performance at a low precursor concentration, about $5 \mathrm{mg} /$ $\mathrm{mL}$, which is markedly less than the $>80 \mathrm{mg} / \mathrm{mL}$ necessary for
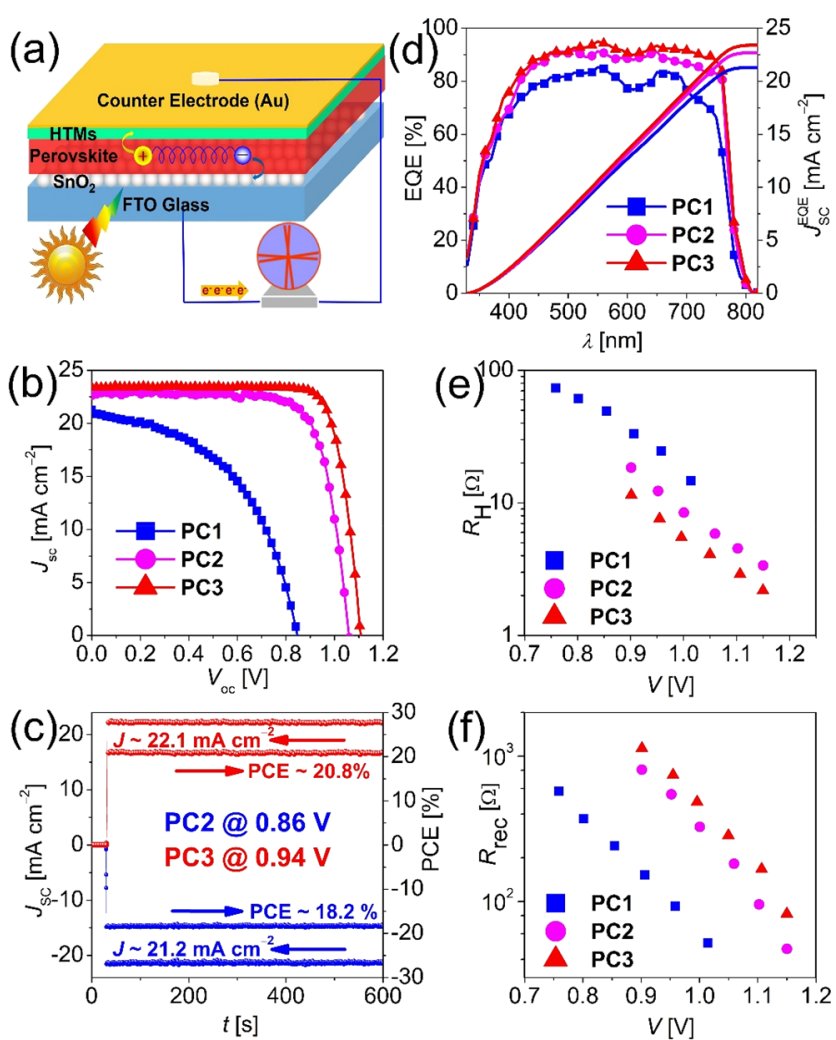

Figure 7. (a) Schematic structure of the PSCs studied. (b) External quantum efficiencies (EQEs) and the corresponding $J_{\mathrm{SC}}^{\mathrm{EQE}}$ derived from the PC1-, PC2-, and PC3-based PSC devices. (c) J-V curves obtained under irradiance of $100 \mathrm{~mW} \mathrm{~cm}^{-2}$. (d) Steady-state output of photocurrent density and PCE recorded at the maximum power point (MPP) under AM 1.5G illumination. (e) Potential biasdependent hole transport resistance $\left(R_{\mathrm{H}}\right)$ of the PSCs. (f) Potential bias-dependent interface recombination resistance $\left(R_{\mathrm{REC}}\right)$ of the PSCs.

spiro-OMeTAD. The perfect coverage of the perovskite surface at the low concentration of the HTMs can be ascribed to the excellent film-forming ability of PC polymers, which in turn can be attributed to the planar backbone-induced strong interchain coupling $(\pi-\pi$ stacking) between adjacent polymer strands. The resistance and electrochemical impedance spectra of PC3 films of different thicknesses (Figure S11b and S11c) demonstrate that the higher resistance of the thicker films (10 $\mathrm{mg} / \mathrm{mL}$ precursor solution concentration) is almost twice as high as that for the thinner films $(5 \mathrm{mg} / \mathrm{mL})$, which most likely will be responsible for the lower FF exhibited by the PSCs based on the thicker films. The low polymer amounts necessary will result in low-cost PSCs when taking the scaling of HTMs into consideration (Figures S12-S16, Tables S5S7). Several key intermediates during the synthesis of PC3 are not commercially available at this moment, which will inevitably push the human resource and materials costs up. However, the significant cost advantage for per cell (low concentration, dopant free, etc.) with respect to that of spiroOMeTAD still qualifies PC3 as a promising candidate for large-scale production of PSCs. Figure $7 \mathrm{~b}$ shows the optimized $J-V$ curves by reverse scanning, and detailed photovoltaic parameters are collected in Table 1. PSCs based on the PC1 HTM display an inferior PCE of $8.8 \%$ with a relatively good short-circuit current density, $J_{\mathrm{SC}}$, of $21.3 \mathrm{~mA} \cdot \mathrm{cm}^{-2}$. However, the PSCs also show a low open-circuit voltage, $V_{\mathrm{OC}}$, of $0.85 \mathrm{~V}$ 
Table 1. Photovoltaic Parameters of Champion PSCs Based on Dopant-Free Polymeric HTMs and Doped Spiro-OMeTAD ${ }^{a}$

\begin{tabular}{|c|c|c|c|c|c|}
\hline HTMs & $J_{\mathrm{SC}}^{\mathrm{EQE}}\left[\mathrm{mA} \mathrm{cm}^{-2}\right]$ & $J_{\mathrm{SC}}\left[\mathrm{mA} \mathrm{cm}{ }^{-2}\right]$ & $V_{\mathrm{OC}}[\mathrm{V}]$ & $\mathrm{FF}[\%]$ & PCE $[\%]$ \\
\hline PC1 & 21.1 & 21.3 & 0.85 & 49.1 & 8.8 \\
\hline PC2 & 22.6 & 22.7 & 1.06 & 76.2 & 18.3 \\
\hline PC3 & 23.5 & 23.5 & 1.11 & 80.0 & 20.8 \\
\hline Spiro-OMeTAD & 23.2 & 23.3 & 1.10 & 78.8 & 20.3 (doped) \\
\hline
\end{tabular}

and fill factor, FF, of $49.1 \%$ along with a problematic hysteresis effect (Figure S17). Obviously, the low hole mobility of PC1 manifests itself in the inefficient PSCs. In contrast, after configurational tuning of the polymer backbone structure, PC2-based PSCs demonstrate much better performance with a good PCE of $18.3 \%$ together with a $J_{\mathrm{SC}}$ of $22.7 \mathrm{~mA} \cdot \mathrm{cm}^{-2}$, a $V_{\mathrm{OC}}$ of $1.06 \mathrm{~V}$, and a FF of $76.2 \%$. Meanwhile, no obvious hysteresis effect can be observed for PC2-based PSCs. As shown above, inclusion of Se increased the hole mobility of PC3 as well as improved the passivation of the perovskite surface. As a result, a better PCE of $20.8 \%$ was displayed by PC3-based PSCs with negligible hysteresis, generating an excellent $J_{\mathrm{SC}}$ of $23.5 \mathrm{~mA} \cdot \mathrm{cm}^{-2}, V_{\mathrm{OC}}$ of $1.11 \mathrm{~V}$, and $\mathrm{FF}$ of $80.0 \%$, ranking this type of PSC as one of the best reported by employing dopant-free HTMs. The PC3-based PSCs are superior to the reference one including doped spiro-OMeTAD as HTM (Figure S18). Furthermore, the power output at the maximum power point (MPP) was recorded for $1500 \mathrm{~s}$, and highly stable PCEs of $18.2 \%$ and $20.8 \%$ are obtained for PC2and PC3-based PSCs, respectively (Figure 7c). It is notable that all of the photovoltaic results are in good agreement with the structure-based analysis and prediction above. As shown in Figure $7 d$, external quantum efficiencies (EQEs) were obtained for the PSCs based on the three dopant-free HTMs as a function of wavelength. PC2- and PC3-based PSCs both show excellent EQEs in a broad range from 400 to $800 \mathrm{~nm}$, generating integrated $J_{\mathrm{SC}}^{\mathrm{EQ}}$ of 22.6 and $23.5 \mathrm{~mA} \cdot \mathrm{cm}^{-2}$, respectively. Nevertheless, The $J_{\mathrm{SC}}^{\mathrm{EQE}}$ of $21.1 \mathrm{~mA} \cdot \mathrm{cm}^{-2}$ for PC1-based PSCs is much lower than those of PC2- and PC3based devices, which should be ascribed to its poor charge extraction properties. All of the $J_{\mathrm{SC}}^{\mathrm{EQE}}$ calculated from the EQEs are in good accordance with the $J_{S C}$ depicted in Figure $7 \mathrm{~b}$.

From the cross-sectional SEM image of PC3-based PSCs, a ca. $40 \mathrm{~nm}$ thick HTM layer can be observed, showing the uniform and complete coverage of the surface of the $\sim 450 \mathrm{~nm}$ thick perovskite layer (Figure S19). For PC1, the performance of the resulting PSCs is significantly lower as the concentration of HTM precursors is increased from 5 to $10 \mathrm{mg} / \mathrm{mL}$, especially regarding the FF and $V_{\mathrm{OC}}$. Most likely a thicker film suffers from the charge transport losses due to the intrinsically low conductivity of the organic materials. However, PC3-based PSCs exhibit comparable PCEs independent of the precursor concentration changes, which should be attributed to the high hole mobility of PC3. The PCEs independent of the HTM thickness are crucial for the conformity rate in future scalable roll-to-roll printing production. A lower concentration of HTM precursors than $5 \mathrm{mg} / \mathrm{mL}$ will give rise to incomplete coverage of the perovskite surface, resulting in severe shunt loss at the interface. We carried out a statistical analysis of 30 devices for PSCs based on each polymer to monitor the reproducibility of PC1-, PC2-, and PC3-based PSCs. As displayed in Figure S20, the distribution of the median PCE gets more narrow going from PC1- to PC3-based PSCs, probably reflecting a hole mobility dependency. In order to track the synthetic reproducibility of PC3, three different batches were synthesized and applied in PSCs. As shown in Figure S21, the statistic PCEs of PSCs suggest excellent synthetic reproducibility and device reproducibility of PC3, extricating PC3-based PSCs from vulnerable device fabrication, especially for large-scale production. In order to gain insights into the origin of $V_{\mathrm{OC}}$ and FF variations, we employed electrochemical impedance spectroscopy (EIS) at a range of potential bias. A simplified equivalent circuit model used for analysis is presented in the Supporting Information. The model unveiled two key parameters, $R_{\mathrm{H}}$ and $R_{\text {rec }}{ }^{75}$ where $R_{\mathrm{H}}$ represents the transport resistance of the hole-transport layer and $R_{\mathrm{rec}}$ is determined by the charge recombination resistance at the HTM/perovskite interface. As shown in Figure 7e, the PSCs of PC1, PC2, and PC3 exhibit a stepwise lower $R_{\mathrm{H}}$ at a given potential bias, which can be ascribed to the stepwise increase of thin film hole mobility. At the same potential bias, an incremental $R_{\text {rec }}$ going from PC1 to PC3 can be observed (Figure 7f), indicating a retarded interfacial charge recombination for PC3-based PSCs. Overall, the higher $R_{\text {rec }}$ and lower $R_{\mathrm{H}}$ agree well with the observed higher $V_{\mathrm{OC}}$ and higher FF for PC3-based PSCs. The $V_{\text {OC }}$ trend of $\mathbf{P C 1}>\mathbf{P C 2}>\mathbf{P C} 3$ will be affected by a multitude of effects, such as the quality of coverage of the perovskite film by the polymers and the density of trap states on the surface of the perovskite film. The low $V_{\mathrm{OC}}$ of PC1-based devices is mainly caused by the poor coverage of the perovskite film by PC1 (Figure S8). The higher $V_{\mathrm{OC}}$ of PC3-based devices, with respect to that of PC2, can be attributed to the strong passivation effects of selenium reducing the density of the trap state sites on the surface of the perovskite film. Therefore, it is reasonable that the lower densities of the trap states (Figure $5 b)$ cause the interface recombination resistances to become higher (Figure 7f) going from PC1 to PC3.

Moisture-induced decomposition of the perovskite film is a critical degradation path for PSCs. Consequently, in our rational design of the PC-based polymeric HTMs, a number of bulky and hydrophobic aliphatic chains were introduced to improve the water-repelling properties of the resulting HTMcoated perovskite films. In general, the alkyl groups usually disturb the interaction between the HTMs and the perovskite films and thus hinder efficient charge transfer. However, in the current systems, a delicate balance was established to maintain excellent humidity resistivity on the premise of a highly strong interface interaction by introducing the PC units as well as selenium in the polymer candidates. Quite large contact angles $\left(\sim 100^{\circ}\right)$ with respect to water droplets were obtained by the PC-based polymers when applied as capping layers with respect to the neat perovskite film $\left(\sim 60^{\circ}\right)$. This indicates an excellent protection of the hygroscopic perovskite material from moisture by the PC-based polymers (Figure S22). The device stability of our champion PSCs containing PC3 as the dopant-free HTM was investigated and compared to a device based on the well-known spiro-OMeTAD (Figure S23). The devices were first placed under ambient atmosphere with 


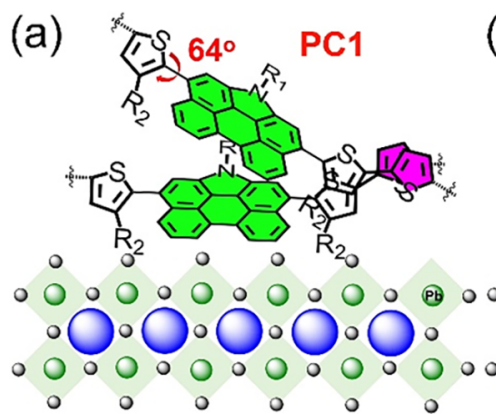

(b)

(c) $\mathrm{PC} 3$

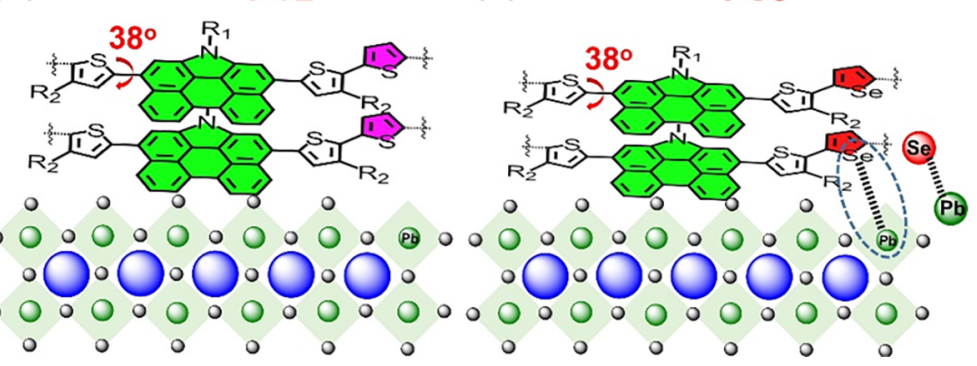

Figure 8. Schematic stacking models of the polymers including the perovskite surface: (a) PC1, (b) PC2, and (c) PC3.

indoor light. The PSCs capped with PC3 as dopant-free HTM layer maintained a nearly unaltered PCE after $1000 \mathrm{~h}$ with respect to the original one. In contrast, the control PSCs coated by doped spiro-OMeTAD suffered a dramatic decrease of PCE during the test. Subsequently, the stability of the PSCs was further evaluated under continuous AM1.5 G equivalent light irradiation at 40 and $85{ }^{\circ} \mathrm{C}$. After a fast initial burn-in decay, likely involving migration of the A-site cations in the perovskite, $^{29}$ the PC3-based PSCs remained highly stable at 40 ${ }^{\circ} \mathrm{C}$. However, the control PSCs based on doped spiroOMeTAD display a $>80 \%$ PCE loss after $1000 \mathrm{~h}$. This should be attributed to the hygroscopic dopants FK209, TBP, and LiTFSI used in combination with spiro-OMeTAD, which will definitely accelerate the decomposition of perovskite films involving water. The obvious PCE decrease of PSCs at $85{ }^{\circ} \mathrm{C}$ should be attributed to the insufficient thermal stability of perovskite materials. The stability test highlights the remarkable ability of the dopant-free polymers studied in this work to generate excellent photovoltaic performance and excellent durability of the resulting PSC devices.

Figure 8 displays schematic stacking models of PC-based polymers and the interaction with a perovskite surface, giving us an image for rational design of dopant-free polymeric HTMs. Despite well-known prerequisites, (1) a well-aligned HOMO energy level with respect to the perovskite valence band (VB) to extract holes in near unity yield, (2) significantly higher LUMO energy level than the perovskite conduction band bottom (CB) to obstruct contrary electron flow, (3) good hole mobility for efficient charge extraction and transport, and (4) excellent thermal and optical phase stability, several guiding design principles directly correlated to the molecular structure have also been highlighted in this work. First, a large conjugated system with a rigid and planar backbone can be inserted into the polymer chains to enhance the $\pi-\pi$ stacking of adjacent polymer strands and also with the perovskite surface. Second, the suitable dihedral angles should be addressed between the broad backbone and connected units. A too large dihedral angle may result in disordered packing of the polymers strands. This may, from a design point of view, be a difficult balance since a too small dihedral angle may induce low solubility and therefore become an obstacle for solution processing. Third, several bulky and hydrophobic alkyl chains can be grafted on the polymeric backbone to improve the humidity resistivity and solubility of polymer provided that strong $\pi-\pi$ stacking is not prevented. Finally, several "Lewis soft" atoms, characteristic of being highly electron-rich and having a large atomic radius, can be introduced as efficient passivators to bond to coordinatively unsaturated lead ions at the surface of the perovskite layer and to enhance the interfacial charge transfer.

\section{CONCLUSION}

To summarize, we synthesized a series of novel and costeffective polymeric semiconductors PC1, PC2, and PC3 characterized by a rigid PC plane and explored their application as dopant-free HTMs in PSCs. Introduction of a broad and planar PC unit significantly enhanced the $\pi-\pi$ stacking of adjacent polymer strands as well as the interaction with the perovskite surface. The configurational control of PC1 mitigates the huge steric hindrance between combined units and generates a more planar geometry as realized in PC2, promoting a face-on-oriented stacking and good charge transport properties. Subsequently, compositional tuning by introducing the "Lewis soft" selenium element improved passivation of the trap-state defects at the PC polymer/ perovskite interface and thereby reduced recombination losses. As a consequence, the PSCs based on the well-designed PC3 gave rise to an excellent PCE of $20.8 \%$, ranking as one of the best performances recorded by PSCs based on dopant-free HTMs. Benefiting from the very strong polymer strand stacking promoted by the PC units, several bulky and hydrophobic aliphatic chains can be grafted on the polymer backbone without restraining interstrand orbital overlap and thereby hole mobility. The excellent humidity resistivity of the polymers resulted in excellent PSC device durability and morphology stability under the dual pressure of thermal stress and light soaking. The combination of excellent PCE and longterm stability for PSCs based on the newly designed PC3 polymer offer an encouraging platform for commercially feasible PSCs. Several guiding principles for further rational design of dopant-free polymeric HTMs are also revealed in this work.

\section{ASSOCIATED CONTENT}

\section{Supporting Information}

The Supporting Information is available free of charge at https://pubs.acs.org/doi/10.1021/jacs.0c08352.

Detailed description of the experimental methods, including molecular synthesis, film deposition, device fabrication and characterization, additional data and figures, and NMR spectra (PDF)

\section{AUTHOR INFORMATION}

\section{Corresponding Author}

Licheng Sun - Department of Chemistry, Organic Chemistry, KTH Royal Institute of Technology, Stockholm 10044, Sweden; State Key Laboratory of Fine Chemicals, Institute of Artificial 
Photosynthesis, DUT-KTH Joint Education and Research Centre on Molecular Devices, Dalian University of Technology (DUT), Dalian 116024, China; Center of Artificial Photosynthesis for Solar Fuels, School of Science, Westlake University, Hangzhou 310024, China; 이이. org/00000002-4521-2870; Email: lichengs@kth.se

\section{Authors}

Zhaoyang Yao - Department of Chemistry, Organic Chemistry, KTH Royal Institute of Technology, Stockholm 10044, Sweden

Fuguo Zhang - Department of Chemistry, Organic Chemistry, KTH Royal Institute of Technology, Stockholm 10044, Sweden; (1) orcid.org/0000-0002-2789-7714

Yaxiao Guo - Department of Chemistry, Organic Chemistry, KTH Royal Institute of Technology, Stockholm 10044, Sweden

Heng Wu - Department of Chemistry, Zhejiang University, Hangzhou 310028, China

Lanlan He - Department of Chemistry, Applied Physical Chemistry, KTH Royal Institute of Technology, Stockholm 10044, Sweden

Zhou Liu - School of Physical Science and Technology, ShanghaiTech University, Shanghai 201210, China

Bin Cai - State Key Laboratory of Fine Chemicals, Institute of Artificial Photosynthesis, DUT-KTH Joint Education and Research Centre on Molecular Devices, Dalian University of Technology (DUT), Dalian 116024, China

Yu Guo - Department of Chemistry, Applied Physical Chemistry, KTH Royal Institute of Technology, Stockholm 10044, Sweden

Calvin J. Brett - Deutsches Elektronen-Synchrotron (DESY), Hamburg 22607, Germany; Department of Engineering Mechanics and Wallenberg Wood Science Center, KTH Royal Institute of Technology, Stockholm 10044, Sweden; (1) orcid.org/0000-0001-5789-6299

Yuanyuan Li - Wallenberg Wood Science Center, Department of Fibre and Polymer Technology, KTH Royal Institute of Technology, Stockholm 10044, Sweden; 이 orcid.org/00000002-1591-5815

Chinmaya Venugopal Srambickal - Experimental Biomolecular Physics, Department of Applied Physics, KTH Royal Institute of Technology, Stockholm SE-10691, Sweden

Xichuan Yang - State Key Laboratory of Fine Chemicals, Institute of Artificial Photosynthesis, DUT-KTH Joint Education and Research Centre on Molecular Devices, Dalian University of Technology (DUT), Dalian 116024, China; (1) orcid.org/0000-0002-3035-4163

Gang Chen - School of Physical Science and Technology, ShanghaiTech University, Shanghai 201210, China; (1) orcid.org/0000-0001-8112-3579

Jerker Widengren - Experimental Biomolecular Physics, Department of Applied Physics, KTH Royal Institute of Technology, Stockholm SE-10691, Sweden; 이이.org/00000003-3200-0374

Dianyi Liu - School of Engineering, Westlake University, Hangzhou 310024, China

James M. Gardner - Department of Chemistry, Applied Physical Chemistry, KTH Royal Institute of Technology, Stockholm 10044, Sweden; 이이. orcid.org/0000-0002-47824969

Lars Kloo - Department of Chemistry, Applied Physical Chemistry, KTH Royal Institute of Technology, Stockholm 10044, Sweden; (1) orcid.org/0000-0002-0168-2942

Complete contact information is available at: https://pubs.acs.org/10.1021/jacs.0c08352

\section{Author Contributions}

${ }^{+}$Z.Y., F. Z., and Y.G.: These authors contributed equally. Notes

The authors declare no competing financial interest.

\section{ACKNOWLEDGMENTS}

This work was financially supported by the Swedish Energy Agency, the Swedish Research Council, the Knut and Alice Wallenberg Foundation, the Swedish government by STandUP for ENERGY and the Swedish Strategic Research FoundationSSF (RAM15-0130), and the National Natural Science Foundation of China (21120102036 and 91233201). We thank the National Supercomputer Centre at Linköping University for computation resources.

\section{REFERENCES}

(1) Kojima, A.; Teshima, K.; Shirai, Y.; Miyasaka, T. Organometal Halide Perovskites as Visible-Light Sensitizers for Photovoltaic Cells. J. Am. Chem. Soc. 2009, 131 (17), 6050-6051.

(2) Kim, H.-S.; Lee, C.-R.; Im, J.-H.; Lee, K.-B.; Moehl, T.; Marchioro, A.; Moon, S.-J.; Humphry-Baker, R.; Yum, J.-H.; Moser, J. E.; Grätzel, M.; Park, N.-G. Lead Iodide Perovskite Sensitized AllSolid-State Submicron Thin Film Mesoscopic Solar Cell with Efficiency Exceeding 9\%. Sci. Rep. 2012, 2 (1), 591.

(3) Lee, M. M.; Teuscher, J.; Miyasaka, T.; Murakami, T. N.; Snaith, H. J. Efficient Hybrid Solar Cells Based on Meso-Superstructured Organometal Halide Perovskites. Science 2012, 338 (6107), 643-647.

(4) Yang, W. S.; Park, B.-W.; Jung, E. H.; Jeon, N. J.; Kim, Y. C.; Lee, D. U.; Shin, S. S.; Seo, J.; Kim, E. K.; Noh, J. H.; Seok, S. I. Iodide Management in Formamidinium-Lead-Halide-Based Perovskite Layers for Efficient Solar Cells. Science 2017, 356 (6345), 1376-1379.

(5) Jeon, N. J.; Na, H.; Jung, E. H.; Yang, T.-Y.; Lee, Y. G.; Kim, G.; Shin, H.-W.; Il Seok, S.; Lee, J.; Seo, J. A Fluorene-Terminated HoleTransporting Material for Highly Efficient and Stable Perovskite Solar Cells. Nat. Energy 2018, 3 (8), 682-689.

(6) Jung, E. H.; Jeon, N. J.; Park, E. Y.; Moon, C. S.; Shin, T. J.; Yang, T.-Y.; Noh, J. H.; Seo, J. Efficient, Stable and Scalable Perovskite Solar Cells Using Poly(3-hexylthiophene). Nature 2019, 567 (7749), 511-515.

(7) Jiang, Q.; Zhao, Y.; Zhang, X.; Yang, X.; Chen, Y.; Chu, Z.; Ye, Q.; Li, X.; Yin, Z.; You, J. Surface Passivation of Perovskite Film for Efficient Solar Cells. Nat. Photonics 2019, 13 (7), 460-466.

(8) Jena, A. K.; Kulkarni, A.; Miyasaka, T. Halide Perovskite Photovoltaics: Background, Status, and Future Prospects. Chem. Rev. 2019, 119 (5), 3036-3103.

(9) NREL Best Research-cell Efficiencies Chart; https://www.nrel. gov/pv/cell-efficiency.html.

(10) Stranks, S. D.; Eperon, G. E.; Grancini, G.; Menelaou, C.; Alcocer, M. J. P.; Leijtens, T.; Herz, L. M.; Petrozza, A.; Snaith, H. J. Electron-Hole Diffusion Lengths Exceeding 1 Micrometer in an Organometal Trihalide Perovskite Absorber. Science 2013, 342 (6156), 341-344.

(11) Tong, J.; Song, Z.; Kim, D. H.; Chen, X.; Chen, C.; Palmstrom, A. F.; Ndione, P. F.; Reese, M. O.; Dunfield, S. P.; Reid, O. G.; Liu, J.; Zhang, F.; Harvey, S. P.; Li, Z.; Christensen, S. T.; Teeter, G.; Zhao, D.; Al-Jassim, M. M.; van Hest, M. F. A. M.; Beard, M. C.; Shaheen, S. E.; Berry, J. J.; Yan, Y.; Zhu, K. Carrier Lifetimes of $>1 \mu$ s in $\mathrm{Sn}-\mathrm{Pb}$ Perovskites Enable Efficient All-Perovskite Tandem Solar Cells. Science 2019, 364 (6439), 475-479.

(12) Dong, Q.; Fang, Y.; Shao, Y.; Mulligan, P.; Qiu, J.; Cao, L.; Huang, J. Electron-Hole Diffusion Lengths $>175 \mu \mathrm{m}$ in SolutionGrown $\mathrm{CH}_{3} \mathrm{NH}_{3} \mathrm{PbI}_{3}$ Single Crystals. Science 2015, 347 (6225), 967970

(13) Eames, C.; Frost, J. M.; Barnes, P. R. F.; O’Regan, B. C.; Walsh, A.; Islam, M. S. Ionic Transport in Hybrid Lead Iodide Perovskite Solar Cells. Nat. Commun. 2015, 6 (1), 7497. 
(14) Wang, R.; Mujahid, M.; Duan, Y.; Wang, Z.-K.; Xue, J.; Yang, Y. A Review of Perovskites Solar Cell Stability. Adv. Funct. Mater. 2019, 29 (47), 1808843.

(15) Wang, Q.; Phung, N.; Di Girolamo, D.; Vivo, P.; Abate, A. Enhancement in Lifespan of Halide Perovskite Solar Cells. Energy Environ. Sci. 2019, 12 (3), 865-886.

(16) Zhang, F.; Yang, X.; Cheng, M.; Wang, W.; Sun, L. Boosting the Efficiency and the Stability of Low Cost Perovskite Solar Cells by Using Cupc Nanorods as Hole Transport Material and Carbon as Counter Electrode. Nano Energy 2016, 20, 108-116.

(17) Park, N.-G.; Grätzel, M.; Miyasaka, T.; Zhu, K.; Emery, K. Towards Stable and Commercially Available Perovskite Solar Cells. Nat. Energy 2016, 1 (11), 16152.

(18) Lai, H.; Kan, B.; Liu, T.; Zheng, N.; Xie, Z.; Zhou, T.; Wan, X.; Zhang, X.; Liu, Y.; Chen, Y. Two-Dimensional Ruddlesden-Popper Perovskite with Nanorod-Like Morphology for Solar Cells with Efficiency Exceeding 15\%. J. Am. Chem. Soc. 2018, 140 (37), 1163911646.

(19) Roose, B.; Wang, Q.; Abate, A. The Role of Charge Selective Contacts in Perovskite Solar Cell Stability. Adv. Energy Mater. 2018, 9 (5), 1803140.

(20) Han, T.-H.; Tan, S.; Xue, J.; Meng, L.; Lee, J.-W.; Yang, Y. Interface and Defect Engineering for Metal Halide Perovskite Optoelectronic Devices. Adv. Mater. 2019, 31 (47), 1803515.

(21) Grancini, G.; Nazeeruddin, M. K. Dimensional Tailoring of Hybrid Perovskites for Photovoltaics. Nature Reviews Materials 2019, 4 (1), 4-22.

(22) Uddin, A.; Upama, M. B.; Yi, H.; Duan, L. Encapsulation of Organic and Perovskite Solar Cells: A Review. Coatings 2019, 9 (2), 65 .

(23) Cheng, M.; Chen, C.; Yang, X.; Huang, J.; Zhang, F.; Xu, B.; Sun, L. Novel Small Molecular Materials Based on Phenoxazine Core Unit for Efficient Bulk Heterojunction Organic Solar Cells and Perovskite Solar Cells. Chem. Mater. 2015, 27 (5), 1808-1814.

(24) Chen, H.; Bryant, D.; Troughton, J.; Kirkus, M.; Neophytou, M.; Miao, X.; Durrant, J. R.; McCulloch, I. One-Step Facile Synthesis of a Simple Hole Transport Material for Efficient Perovskite Solar Cells. Chem. Mater. 2016, 28 (8), 2515-2518.

(25) Saliba, M.; Orlandi, S.; Matsui, T.; Aghazada, S.; Cavazzini, M.; Correa-Baena, J.-P.; Gao, P.; Scopelliti, R.; Mosconi, E.; Dahmen, K.H.; De Angelis, F.; Abate, A.; Hagfeldt, A.; Pozzi, G.; Graetzel, M.; Nazeeruddin, M. K. A Molecularly Engineered Hole-Transporting Material for Efficient Perovskite Solar Cells. Nat. Energy 2016, 1 (2), 15017.

(26) Xu, B.; Zhang, J.; Hua, Y.; Liu, P.; Wang, L.; Ruan, C.; Li, Y.; Boschloo, G.; Johansson, E. M. J.; Kloo, L.; Hagfeldt, A.; Jen, A. K. Y.; Sun, L. Tailor-Making Low-Cost Spiro[fluorene-9,9'-xanthene]-Based 3D Oligomers for Perovskite Solar Cells. Chem. 2017, 2 (5), 676687.

(27) Ge, Q.-Q.; Shao, J.-Y.; Ding, J.; Deng, L.-Y.; Zhou, W.-K.; Chen, Y.-X.; Ma, J.-Y.; Wan, L.-J.; Yao, J.; Hu, J.-S.; Zhong, Y.-W. A Two-Dimensional Hole-Transporting Material for High-Performance Perovskite Solar Cells with 20\% Average Efficiency. Angew. Chem., Int. Ed. 2018, 57 (34), 10959-10965.

(28) Chiykowski, V. A.; Cao, Y.; Tan, H.; Tabor, D. P.; Sargent, E. H.; Aspuru-Guzik, A.; Berlinguette, C. P. Precise Control of Thermal and Redox Properties of Organic Hole-Transport Materials. Angew. Chem., Int. Ed. 2018, 57 (47), 15529-15533.

(29) Christians, J. A.; Schulz, P.; Tinkham, J. S.; Schloemer, T. H.; Harvey, S. P.; Tremolet de Villers, B. J.; Sellinger, A.; Berry, J. J.; Luther, J. M. Tailored Interfaces of Unencapsulated Perovskite Solar Cells for > 1,000 h Operational Stability. Nat. Energy 2018, 3 (1), 6874.

(30) Vaitukaityte, D.; Wang, Z.; Malinauskas, T.; Magomedov, A.; Bubniene, G.; Jankauskas, V.; Getautis, V.; Snaith, H. J. Efficient and Stable Perovskite Solar Cells Using Low-Cost Aniline-Based Enamine Hole-Transporting Materials. Adv. Mater. 2018, 30 (45), 1803735.

(31) Shen, C.; Wu, Y.; Zhang, H.; Li, E.; Zhang, W.; Xu, X.; Wu, W.; Tian, H.; Zhu, W.-H. Semi-Locked Tetrathienylethene as a Building
Block for Hole-Transporting Materials: Toward Efficient and Stable Perovskite Solar Cells. Angew. Chem., Int. Ed. 2019, 58 (12), 37843789.

(32) Salbeck, J.; Yu, N.; Bauer, J.; Weissörtel, F.; Bestgen, H. Low Molecular Organic Glasses for Blue Electroluminescence. Synth. Met. 1997, 91 (1), 209-215.

(33) Bach, U.; Lupo, D.; Comte, P.; Moser, J. E.; Weissörtel, F.; Salbeck, J.; Spreitzer, H.; Grätzel, M. Solid-State Dye-Sensitized Mesoporous $\mathrm{TiO}_{2}$ Solar Cells with High Photon-to-Electron Conversion Efficiencies. Nature 1998, 395 (6702), 583-585.

(34) Kim, M.; Kim, G.-H.; Lee, T. K.; Choi, I. W.; Choi, H. W.; Jo, Y.; Yoon, Y. J.; Kim, J. W.; Lee, J.; Huh, D.; Lee, H.; Kwak, S. K.; Kim, J. Y.; Kim, D. S. Methylammonium Chloride Induces Intermediate Phase Stabilization for Efficient Perovskite Solar Cells. Joule 2019, 3 (9), 2179-2192.

(35) Noh, J. H.; Jeon, N. J.; Choi, Y. C.; Nazeeruddin, M. K.; Grätzel, M.; Seok, S. I. Nanostructured $\mathrm{TiO}_{2} / \mathrm{CH}_{3} \mathrm{NH}_{3} \mathrm{PBbI}_{3}$ Heterojunction Solar Cells Employing Spiro-OMeTAD/Co-Complex as Hole-Transporting Material. J. Mater. Chem. A 2013, 1 (38), $11842-11847$.

(36) Abate, A.; Leijtens, T.; Pathak, S.; Teuscher, J.; Avolio, R.; Errico, M. E.; Kirkpatrik, J.; Ball, J. M.; Docampo, P.; McPherson, I.; Snaith, H. J. Lithium Salts as "Redox Active" p-Type Dopants for Organic Semiconductors and Their Impact in Solid-State DyeSensitized Solar Cells. Phys. Chem. Chem. Phys. 2013, 15 (7), 25722579.

(37) Burschka, J.; Dualeh, A.; Kessler, F.; Baranoff, E.; Cevey-Ha, N.-L.; Yi, C.; Nazeeruddin, M. K.; Grätzel, M. Tris(2-(1H-pyrazol-1yl)pyridine)cobalt(III) as p-Type Dopant for Organic Semiconductors and Its Application in Highly Efficient Solid-State Dye-Sensitized Solar Cells. J. Am. Chem. Soc. 2011, 133 (45), 18042-18045.

(38) Leijtens, T.; Lim, J.; Teuscher, J.; Park, T.; Snaith, H. J. Charge Density Dependent Mobility of Organic Hole-Transporters and Mesoporous $\mathrm{TiO}_{2}$ Determined by Transient Mobility Spectroscopy: Implications to Dye-Sensitized and Organic Solar Cells. Adv. Mater. 2013, 25 (23), 3227-3233.

(39) Fabregat-Santiago, F.; Bisquert, J.; Cevey, L.; Chen, P.; Wang, M.; Zakeeruddin, S. M.; Grätzel, M. Electron Transport and Recombination in Solid-State Dye Solar Cell with Spiro-OMeTAD as Hole Conductor. J. Am. Chem. Soc. 2009, 131 (2), 558-562.

(40) Liu, J.; Wu, Y.; Qin, C.; Yang, X.; Yasuda, T.; Islam, A.; Zhang, K.; Peng, W.; Chen, W.; Han, L. A Dopant-Free Hole-Transporting Material for Efficient and Stable Perovskite Solar Cells. Energy Environ. Sci. 2014, 7 (9), 2963-2967.

(41) Jena, A. K.; Ikegami, M.; Miyasaka, T. Severe Morphological Deformation of Spiro-OMeTAD in $\left(\mathrm{CH}_{3} \mathrm{NH}_{3}\right) \mathrm{PbI}_{3}$ Solar Cells at High Temperature. ACS Energy Lett. 2017, 2 (8), 1760-1761.

(42) Parthasarathy, G.; Shen, C.; Kahn, A.; Forrest, S. R. Lithium Doping of Semiconducting Organic Charge Transport Materials. J. Appl. Phys. 2001, 89 (9), 4986-4992.

(43) Juarez-Perez, E. J.; Leyden, M. R.; Wang, S.; Ono, L. K.; Hawash, Z.; Qi, Y. Role of the Dopants on the Morphological and Transport Properties of Spiro-MeOTAD Hole Transport Layer. Chem. Mater. 2016, 28 (16), 5702-5709.

(44) Rakstys, K.; Paek, S.; Gao, P.; Gratia, P.; Marszalek, T.; Grancini, G.; Cho, K. T.; Genevicius, K.; Jankauskas, V.; Pisula, W.; Nazeeruddin, M. K. Molecular Engineering of Face-On Oriented Dopant-Free Hole Transporting Material for Perovskite Solar Cells with 19\% PCE. J. Mater. Chem. A 2017, 5 (17), 7811-7815.

(45) Xu, B.; Bi, D.; Hua, Y.; Liu, P.; Cheng, M.; Grätzel, M.; Kloo, L.; Hagfeldt, A.; Sun, L. A Low-Cost Spiro[fluorene-9,9'-xanthene]Based Hole Transport Material for Highly Efficient Solid-State DyeSensitized Solar Cells and Perovskite Solar Cells. Energy Environ. Sci. 2016, 9 (3), 873-877.

(46) Zhou, W.; Wen, Z.; Gao, P. Less is More: Dopant-Free Hole Transporting Materials for High-Efficiency Perovskite Solar Cells. Adv. Energy Mater. 2018, 8 (9), 1702512.

(47) Hou, W.; Xiao, Y.; Han, G.; Lin, J.-Y. The Applications of Polymers in Solar Cells: A Review. Polymers 2019, 11 (1), 143. 
(48) Al-Hussein, M.; Herzig, E. M.; Schindler, M.; Löhrer, F.; Palumbiny, C. M.; Wang, W.; Roth, S. V.; Müller-Buschbaum, P. Comparative Study of the Nanomorphology of Spray and Spin Coated PTB7 Polymer: Fullerene Films. Polym. Eng. Sci. 2016, 56 (8), 889-894.

(49) Su, B.; Caller-Guzman, H. A.; Körstgens, V.; Rui, Y.; Yao, Y.; Saxena, N.; Santoro, G.; Roth, S. V.; Müller-Buschbaum, P. Macroscale and Nanoscale Morphology Evolution during in Situ Spray Coating of Titania Films for Perovskite Solar Cells. ACS Appl. Mater. Interfaces 2017, 9 (50), 43724-43732.

(50) Kim, G.-W.; Kang, G.; Kim, J.; Lee, G.-Y.; Kim, H. I.; Pyeon, L.; Lee, J.; Park, T. Dopant-Free Polymeric Hole Transport Materials for Highly Efficient and Stable Perovskite Solar Cells. Energy Environ. Sci. 2016, 9 (7), 2326-2333.

(51) Liao, H.-C.; Tam, T. L. D.; Guo, P.; Wu, Y.; Manley, E. F.; Huang, W.; Zhou, N.; Soe, C. M. M.; Wang, B.; Wasielewski, M. R.; Chen, L. X.; Kanatzidis, M. G.; Facchetti, A.; Chang, R. P. H.; Marks, T. J. Dopant-Free Hole Transporting Polymers for High Efficiency, Environmentally Stable Perovskite Solar Cells. Adv. Energy Mater. 2016, 6 (16), 1600502.

(52) Lee, J.; Malekshahi Byranvand, M.; Kang, G.; Son, S. Y.; Song, S.; Kim, G.-W.; Park, T. Green-Solvent-Processable, Dopant-Free Hole-Transporting Materials for Robust and Efficient Perovskite Solar Cells. J. Am. Chem. Soc. 2017, 139 (35), 12175-12181.

(53) Zhang, L.; Liu, C.; Zhang, J.; Li, X.; Cheng, C.; Tian, Y.; Jen, A. K.-Y.; Xu, B. Intensive Exposure of Functional Rings of a Polymeric Hole-Transporting Material Enables Efficient Perovskite Solar Cells. Adv. Mater. 2018, 30 (39), 1804028.

(54) Cai, B.; Xing, Y.; Yang, Z.; Zhang, W.-H.; Qiu, J. High Performance Hybrid Solar Cells Sensitized by Organolead Halide Perovskites. Energy Environ. Sci. 2013, 6 (5), 1480-1485.

(55) Cai, F.; Cai, J.; Yang, L.; Li, W.; Gurney, R. S.; Yi, H.; Iraqi, A.; Liu, D.; Wang, T. Molecular Engineering of Conjugated Polymers for Efficient Hole Transport and Defect Passivation in Perovskite Solar Cells. Nano Energy 2018, 45, 28-36.

(56) Kim, G.-W.; Lee, J.; Kang, G.; Kim, T.; Park, T. DonorAcceptor Type Dopant-Free, Polymeric Hole Transport Material for Planar Perovskite Solar Cells (19.8\%). Adv. Energy Mater. 2018, 8 (4), 1701935.

(57) Kranthiraja, K.; Gunasekar, K.; Kim, H.; Cho, A.-N.; Park, N.G.; Kim, S.; Kim, B. J.; Nishikubo, R.; Saeki, A.; Song, M.; Jin, S.-H. High-Performance Long-Term-Stable Dopant-Free Perovskite Solar Cells and Additive-Free Organic Solar Cells by Employing Newly Designed Multirole $\pi$-Conjugated Polymers. Adv. Mater. 2017, 29 (23), 1700183.

(58) Liao, S.-H.; Jhuo, H.-J.; Cheng, Y.-S.; Chen, S.-A. Fullerene Derivative-Doped Zinc Oxide Nanofilm as the Cathode of Inverted Polymer Solar Cells with Low-Bandgap Polymer (PTB7-Th) for High Performance. Adv. Mater. 2013, 25 (34), 4766-4771.

(59) Meng, L.; Zhang, Y.; Wan, X.; Li, C.; Zhang, X.; Wang, Y.; Ke, X.; Xiao, Z.; Ding, L.; Xia, R.; Yip, H.-L.; Cao, Y.; Chen, Y. Organic and Solution-Processed Tandem Solar Cells with $17.3 \%$ Efficiency. Science 2018, 361 (6407), 1094-1098.

(60) Zhang, F.; Yao, Z.; Guo, Y.; Li, Y.; Bergstrand, J.; Brett, C. J.; Cai, B.; Hajian, A.; Guo, Y.; Yang, X.; Gardner, J. M.; Widengren, J.; Roth, S. V.; Kloo, L.; Sun, L. Polymeric, Cost-Effective, Dopant-Free Hole Transport Materials for Efficient and Stable Perovskite Solar Cells. J. Am. Chem. Soc. 2019, 141 (50), 19700-19707.

(61) Würthner, F. Perylene Bisimide Dyes as Versatile Building Blocks for Functional Supramolecular Architectures. Chem. Commun. 2004, 14, 1564-1579.

(62) Li, C.; Liu, Z.; Schöneboom, J.; Eickemeyer, F.; Pschirer, N. G.; Erk, P.; Herrmann, A.; Müllen, K. Perylenes as Sensitizers in Hybrid Solar Cells: How Molecular Size Influences Performance. J. Mater. Chem. 2009, 19 (30), 5405-5415.

(63) Yao, Z.; Zhang, M.; Wu, H.; Yang, L.; Li, R.; Wang, P. Donor/ Acceptor Indenoperylene Dye for Highly Efficient Organic DyeSensitized Solar Cells. J. Am. Chem. Soc. 2015, 137 (11), 3799-3802.
(64) Yao, Z.; Wu, H.; Li, Y.; Wang, J.; Zhang, J.; Zhang, M.; Guo, Y.; Wang, P. Dithienopicenocarbazole as the Kernel Module of LowEnergy-Gap Organic Dyes for Efficient Conversion of Sunlight to Electricity. Energy Environ. Sci. 2015, 8 (11), 3192-3197.

(65) Spano, F. C. The Spectral Signatures of Frenkel Polarons in Hand J-Aggregates. Acc. Chem. Res. 2010, 43 (3), 429-439.

(66) Müller-Buschbaum, P. The Active Layer Morphology of Organic Solar Cells Probed with Grazing Incidence Scattering Techniques. Adv. Mater. 2014, 26 (46), 7692-7709.

(67) Wang, R.; Tong, Y.; Wang, K.; Xia, S.; Kentzinger, E.; Soltwedel, O.; Müller-Buschbaum, P.; Frielinghaus, H. Monitoring the Morphological Evolution in Mixed-Dimensional Lead Bromide Perovskite Films with Lamellar-Stacked Perovskite Nanoplatelets. Nanoscale Horiz 2019, 4 (5), 1139-1144.

(68) Guo, X.; Zhou, N.; Lou, S. J.; Smith, J.; Tice, D. B.; Hennek, J. W.; Ortiz, R. P.; Navarrete, J. T. L.; Li, S.; Strzalka, J.; Chen, L. X.; Chang, R. P. H.; Facchetti, A.; Marks, T. J. Polymer Solar Cells with Enhanced Fill Factors. Nat. Photonics 2013, 7 (10), 825-833.

(69) Zhang, Q.; Kelly, M. A.; Bauer, N.; You, W. The Curious Case of Fluorination of Conjugated Polymers for Solar Cells. Acc. Chem. Res. 2017, 50 (9), 2401-2409.

(70) Fang, L.; Zheng, A.; Ren, M.; Xie, X.; Wang, P. Unraveling the Structure-Property Relationship of Molecular Hole-Transporting Materials for Perovskite Solar Cells. ACS Appl. Mater. Interfaces 2019, 11 (42), 39001-39009.

(71) Slater, J. C. Atomic Radii in Crystals. J. Chem. Phys. 1964, 41 (10), 3199-3204.

(72) Clementi, E.; Raimondi, D. L.; Reinhardt, W. P. Atomic Screening Constants from SCF Functions. II. Atoms with 37 to 86 Electrons. J. Chem. Phys. 1967, 47 (4), 1300-1307.

(73) Moulder, J. F. Handbook of X-ray Photoelectron Spectroscopy: A Reference Book of Standard Spectra for Identification and Interpretation of XPS Data; Physical Electronics, 1995; pp 230-232.

(74) Zhang, F.; Cong, J.; Li, Y.; Bergstrand, J.; Liu, H.; Cai, B.; Hajian, A.; Yao, Z.; Wang, L.; Hao, Y.; Yang, X.; Gardner, J. M.; Ågren, H.; Widengren, J.; Kloo, L.; Sun, L. A Facile Route to Grain Morphology Controllable Perovskite Thin Films towards Highly Efficient Perovskite Solar Cells. Nano Energy 2018, 53, 405-414.

(75) Gonzalez-Pedro, V.; Juarez-Perez, E. J.; Arsyad, W.-S.; Barea, E. M.; Fabregat-Santiago, F.; Mora-Sero, I.; Bisquert, J. General Working Principles of $\mathrm{CH}_{3} \mathrm{NH}_{3} \mathrm{PbX}_{3}$ Perovskite Solar Cells. Nano Lett. 2014, $14(2), 888-893$. 